\title{
UEBER DAS SPEKTRUM SEISMISCHER WELLEN, DIE DURCH SPRENGUNGEN ERZEUGT WERDEN
}

\author{
H. MenZeL
}

Dic Gesctzmüssirkeiten des Spektrunus scismischer Wellen, die durch Sprengungen erzeugt werden, sind noch verhälnismässig wenig erforscht. Man weiss, dass das Spektrum der Mintrop-Welle sehr von der Enlfernung des Seismographen rom Sprengpunkte ahhüngig ist. In kleinen Entfernungen ist die vorherrschende Frequenz sehr hoch - oft grösser als 700 $\mathrm{Hz}$ - während diese Welle in sehr grossen Entfermungen eine Frequenz von nur wenigen $\mathrm{H}_{z}$ besitzt. Bei den reflektierten Wellen heohachtet man im allgemeinen Frequenzen, die in einem nicht allzu grossen Bereich um $50 \mathrm{II}_{z}$ liegen. Auch hei diesen Wellen kann man Veränderungen des Spektrums mit der Entfernung und mit der Tiefe der reflektierenden Schichten feststellen. Aus diesen Erfahrungstatsachen folgt, dass nicht nur die Vorgänge hei der Sprengung, sondern auch die hei der Fortpflanzung der Wellen in der Erde die Aushildung des Spektrums der Wellen heeinflussen. Die folgenden therlegungen hefassen sich im Wesentliehen mit einigen Effekten, welche Veränderungen des Spektrums sprengseismischer Wellen zur Folqe haben. Aher auch auf die Auslildung des primären Spektrums durch die Vorgänge bei der Sprengung soll eingegangen werden.

Sucht man nach Erscheinungen, die eine Veränderung des Spektrums seismischer Wellen hewirken, so gerät man nicht durch cinen Mangel an derartigen Effekten in Verlegenheit, sondern eher dadurch, dass es deren zu vicle gilut. Wohl lokannt ist in dieser Hinsicht die Rolle, welche die Ahweichung des von der Welle durchlaufenen Bodens von einem rein elastischen Medium spielt. Es ist aher nicht healssichtigt, diese Dinge hier zu diskutieren. Die Erdkruste wird also im Rahmen dieser Arbeit als ein rein elastischen Medium hehandelt werden. Damit soll allerdings nicht hehauptet werden. dass Viskositï, innere Reilung oder elastische Nachwirkung für die Aushildung der Spektren ohne oder auch nur von geringer Bedeutung seien. Das Ziel dieser Untersuchung ist nicht die vollständige Lösung

* La traduzione in lingua italiana della presente Nota è riportata a pag. 433. 
des Frequenzprohlems, sondern die Vorhereitung dazu. Hier werden nur solche Effekte behandelt werden, die im rein elastischen Medium eine Veründerung des Wellenspektrums hewirken. Im Wesentlichen handelt es sich dahei nur um zwei verschiedene phrsikalische Erscheinungen: 1.) Interferenz. 2.) Energiezerstreuung durch Reflexion, Brechung und Beugung

Die Bedeutung der Interferenz für dic Aushildungr des Spek-

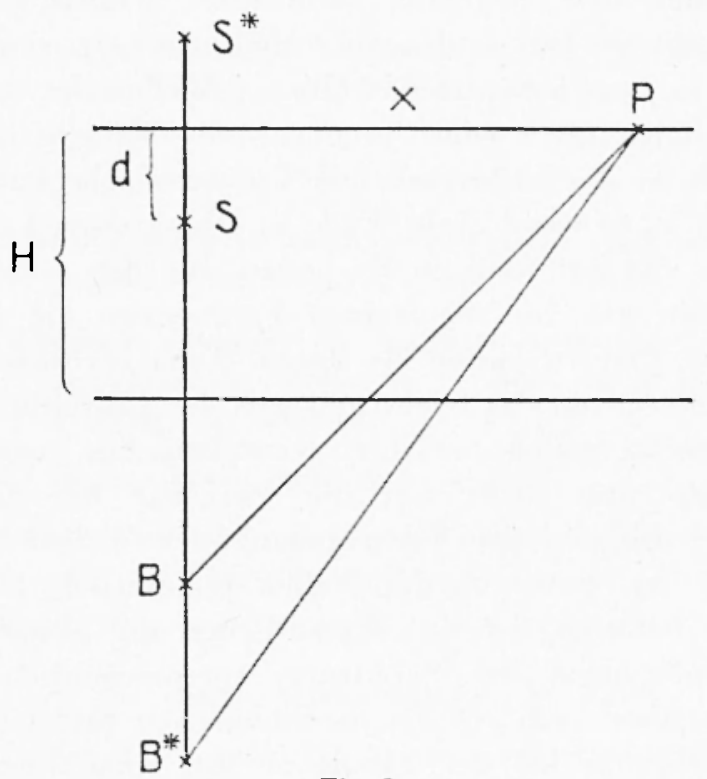

Fig. 1 trums crovilht sich aus dem Unstande, dass die Sprengungen einige Meter unterhall, der Erdoherfläche durchyefuilurt werden. Es interfericren damn in tieferen Untergrunde zwei Wellen: Die diretke durch die Sprengung erzeugrte Welle, und die an der Oherfl̈̈che reflcktierte Welle. Je nach dem Gangunterselied dicser heiden Wellen in cinem hestimmten Punkte werden dort hestimmte Frequenzhereiche des Spektrums verstärkt, andere geschwächt werden. Ninmt man etwa an, dass der Sprengpunkt $15 \mathrm{~m}$ tief unter der Erdoherfläche liegt, und dass die Wellengeschwindigkeit in der ohersten Frdsehicht $1500 \mathrm{~m} / \mathrm{s}$ its, so hetrïgt der Gangunterschied in einem Punkte, der senkrecht unter dem Sprengzentrum liegt, $20 \mathrm{~ms}$. Im Spektrum der Welle wird also der Frequenzhereich um $50 \mathrm{II} \%$ verstïrkt werden. Nun soll der Gangunterschied in einem Punkte $P$ berechnet werden, welcher vom Epizentrum den Alstand $x$ hat (s. Fig. 1.). Die reflektierende Ehene liegre in der Tiefe II und sci parallel der Erdoherflïche. : sei das Sprengzentrum, " $" *$ sein and der Erdoherfl̈̈che gespiegchtes Bild, $B$ dä Bild von ${ }^{C} B^{*}$ das von "**, heide an der reflektierenden Ehene im Untergrunde gespiegrelt. Ist $v$ die Wellengeschwindigkeit, so herechnet man den Gangunterschied in $P$ nach der Formel: 


$$
\Theta=\frac{8 H d}{I_{1}+I_{2}} \frac{1}{v}=4 \frac{\underline{u}}{l} \frac{\underline{d}}{v}
$$

wohei $l$ das Mittel aus $i_{1}$ und ist $l$, ist. Aus Gl. [1] ergilut sich:

1.) Bei festeni $I I$ und $x$ verschieht sich im Spektrum der durch Interferenz verstiirkte Frequenzhereich zu kieineren Werten, wenn die Tiefe des Sprengrpunktes grösser wird.

2.1 Bei festem $d$ und $x$ liegt der verstärkte Frequenzhereich bei kleineren Werten, wenn die reflektierende Ehene tiefer liegrt.

3.) Bei festem $I I$ und $d$ verschiebt sich der verstärkte Frequenzloercich zu grösseren Werten hin, wenn $x$ anwächst.

Rein qualitativ sind diese drei Erscheinungen bei sprengreismischen Beobachtungen tatsichlich festzustellen. Eine quantitative Prïfung, die allein iilser Wert oder Unwert einer Theorie entscheiden $\mathrm{kann}$, ist begonnen worden. Allerdings ist Gl. [1] noch viel zu primitiv, um die wirklichen Verhältnisse quantitativ wiederggehen zu könmen. Eine Verfeinerung hat vor allem dem Einfluss der sog. Verwitterungszone Rechnung zu tragen. Darïher wird später berichtet werden.

Der Hinweis darauf, dass der Frequenzliereich ron etwa $50 \mathrm{~Hz}$ durch Interferenz verstäkt wird, geniigt nicht, um das Vorluerrschen dieser Frequenzen in den reflektierten Wellen zu erklaren. Wenn nämlich dieser Frequenzluereich im primären Spektrum nur eine unterreordnete Rolle spielt, so ist es nicht leicht einzuselıen, wie er durch Interferenz so weit rerstäkt werden kann, dass er schliesslich dominiert. Es ist also eine wichtige Aufgalse, einige Uberlegungen iiber die Verteilung der Frequenzen im primären Spektrum anzustellen. Für dieses Vorhaben liefert eine kürzlich veröflentlichte Arbeit von $H$. Morris (1) den hesten Ansatzpunkt. Morris unterscheidet im Alblauf der Vorgänge nach Auslösen einer Explosion drei Stadien. In ersten, selir rasch verlaufenden Stadium, wird der Sprengstoff in ein selır heisses und hochgespanntes $G$ as verwandelt, olme dass die Wandung des Schusshohrloches merklich nachugiht. In zweiten Stadium loreitet sich cine Stosswelle kugelförnig unter Zertrimmerung des Gesteins in Medium aus, his sie einen kritischen Radius $R$ erreicht. Dieser -t dadurch gekennzeichnet, dass der Maximalhetrag der durch dir Stosswelle im Gestein erzeugten elastischen Spanmung so weit ahgenommen hat, dass der weitere Vorgang -.- das dritte Stadium also nach den Gesetzen der Elastizitätstheorie verläuft. Dieses dritte Stadium soll hier näher betrachtet werden. In einem nach allen Richtungen hin unbegrenzten, homogenen und isotropen elastischen Medium 
sei eine kụ̆celförmige Höhle rom Radius $R$ vorhanden. Auf die Kugrelfliche werde von innen licr ein nach einem beliebigren Zeitgesels W (t) rerlaufender Normaldruck ausgeüht. Es soll die elastische Welle herechnet werden, welche von der Kugelfache ablüuft.

Aus Symmetriegrïinden ist nur cine longitudinale, kugrelsymmetrische Welle möglich. Legrt man den Ursprung eines räumlichen Polarkoordinatensystems in den Mittelpunkt der Hohlkungel, so ist die Lösung des Problems nur von einer Raumkoordinaten, der Länge $r$ des Radiusvektors ahhingrior, und der Vektor $\vec{\sigma}$ der elastischen Verschiehungr hat nur eine Komponente $\sigma_{\mathrm{r}}$ in Richtung des Radiusvektors. Es ist

$$
\vec{\sigma}=\operatorname{grad} F(r, t)
$$

d. h.

$$
\sigma_{\mathrm{r}}=\frac{\partial F}{\partial r}
$$

Aus der Gleichungr des Elastizilätstheorie:

$$
\varrho \frac{\partial^{2} \sigma}{\partial t^{2}}=(\lambda+2 \mu) \text { grad } d i \vec{v} \vec{\sigma}-\mu \operatorname{rot} \operatorname{rot} \vec{\sigma}
$$

folgt dann für $F$ die Differentialgleichungr:

$$
0 \frac{\partial^{2} F}{\partial t^{\circ}}=(\lambda+2 \mu) \backslash \mathrm{F}
$$

Dabei bezeichnet (" die Dichte des Mediums, und 2. und It sind seine Lamésche Konstanten. Die Normalkomponente des Spamnungstensors herechnet man durch:

Das ergịilut :

$$
N=(\lambda+2 \mu) \text { div } \vec{\sigma}-4 \mu \frac{1}{r} \sigma_{r}
$$

$$
\Lambda=(\lambda+2 \imath) \Delta F-4 ! \imath \frac{1}{r} \frac{\partial F}{\partial r}
$$

Zu der Differentialg̣leicloungr [3 a] tritt nun noch die Randlueding̣ung:

$$
(\lambda+2 u) \Delta F-4 ;: \frac{1}{R} \frac{\partial F}{\partial r}+\omega(t)=0 \quad, \quad \text { für } r=R
$$

Aus [3 a] folı̣t die Lösung̣:

$$
F=\frac{1}{r} H(r-v t)
$$


woljei

$$
v=1 \sqrt{\frac{\overline{t+2 u}}{\varrho}}
$$

grilt, und $H$ eine zunächst noch beliehige Funktion ist. Aus [6] und [5] erliält man:

$$
\frac{1+2 \mu}{R} H^{\prime \prime}(R-v t)-\frac{4: v}{R^{2}} H^{\prime}(R-v t)+\frac{4 \underline{\mu}}{R^{3}} H(R-v t)+\Phi(t)=0
$$

Setzt man $R-v t=\S$ und $\Phi(t)=\Psi(\xi)$, so kann man $H$ aus der ge. wönlichen Differentialgleichung

$$
\frac{\lambda+2 u}{R} H^{\prime \prime}(\xi)-\frac{4 u}{R^{2}} H^{\prime}(\xi)+\frac{4 \cdot:}{R^{3}} H(\xi)=-\Psi^{\prime}(\xi) \quad[8 \mathrm{a}]
$$

hestimmen. Die Lösung ist:

$$
H(r-v t)=\frac{i}{4} \frac{h^{2}}{\rho v} \frac{i+2 \mu}{\sqrt{\mu(\hat{\imath}+\mu)}} \int_{0}^{\frac{r-R}{v}}\left[e^{\sigma_{1}\left(t-\frac{r-R}{v}-\tau\right)}-e^{\sigma_{2}\left(t-\frac{r-R}{v}-\tau\right)}\right] \Phi(\tau) d \tau
$$

Die Grössen $\sigma_{1}$ und $\sigma_{0}$ berechnet man aus :

$$
\sigma_{1}=\frac{v}{R}\left(-\frac{2 \mu}{\lambda+2 \mu}+2 i \frac{\overline{u(\lambda+\mu)}}{\lambda+2 \mu}\right), \quad \sigma_{2}=\frac{v}{R}\left(-\frac{2 \mu}{\lambda+2 \mu}-2 i \frac{\overline{l(\lambda+\mu)}}{\lambda+2 \mu}\right)
$$

Das Auftreten der e -Funktion mit komplexem Argument im Integranden von [9] hedeutet, dass die Bodenhewegungr in jedem Punkte einen Anteil enthält, der als eine gedämpfte Eigenselıwingung hezeichnet werden kann. Die Frequenz dieser Eigenschwingung ist:

$$
(1)=2 \frac{v}{R} \sqrt{\frac{\mu(\lambda+u)}{\lambda+2 u}}
$$

und ilure Dämpfungskonstante

$$
\varepsilon=2 \frac{v}{R} \frac{\iota}{\lambda+2 \iota}
$$

Um Gl. [9] näher zu studieren, muss man die Funktion $\Phi(t)$ kennen. Leider weiss man zur Zeit noch nichts Genaues üher diese Funktion, es wird aher eine gewisse Annäherung an die Wirklichkeit darstellen, wenn man mit der Annalıme:

$$
\begin{aligned}
& \text { () }(t)=0 \\
& \text { fi } \mathrm{r}-\infty<t<0 \\
& \text { () }(t)=a t e^{-a t} \\
& u=t<x
\end{aligned}
$$


rechnet. Aus [12], [9], [6] und ([2 a] folgt dann:

$$
\begin{aligned}
& \sigma_{\mathrm{r}}=\frac{a k^{2}}{2 \gamma^{\prime} \overline{(\lambda+\mu)}\left[\left((\iota-\varepsilon)^{2}+\left(\omega^{2}\right]^{2}\right.\right.} \frac{1}{r} . \\
& \left\{\left(\frac{v}{r}-\varepsilon\right)\left[(\alpha-\varepsilon)^{2}-\omega^{2}\right]+2 \omega^{2}(\alpha-\varepsilon)\right\} e^{-\varepsilon\left(t-\frac{r-R}{v}\right)} \sin (1)\left(t-\frac{r-R}{v}\right)+ \\
& +\omega\left[\left((t-\varepsilon)^{2}-\omega^{2}-2\left(\frac{v}{r}-\varepsilon\right)(\alpha-\varepsilon)\right] e^{-\varepsilon\left(t-\frac{r-R}{v}\right)} \cos \omega\left(t-\frac{r-R}{v}\right)-\right. \\
& -\left\{\left(\frac{v}{r}-\varepsilon\right)\left[(\alpha-\varepsilon)^{2}-\omega^{2}\right]+2 \omega^{2}(\alpha-\varepsilon)\right\} \omega\left(t-\frac{r-R}{v}\right) e^{-\alpha\left(t-\frac{r-R}{v}\right)}- \\
& -(1)\left[(\alpha-\varepsilon)^{2}-\left(\omega^{2}-2\left(\frac{v}{r}-\varepsilon\right)(\alpha-\varepsilon)\right]\left[1+(\alpha-\varepsilon)\left(t-\frac{r-R}{v}\right)\right] e^{-\alpha\left(t-\frac{r-R}{v}\right)}\right)
\end{aligned}
$$

Die heiden ersten Terme der Gl. [13] stellen die sich durch das Medium fortpflanzende gedämpte Eigenschwingung, die heiden letzten Terme die Fortpflanzung der Störung dar. Amplitude und Phase der Eigenschwingung sind von $r$ ahhängig. Daher veründert sich das Bilis der Schwingung von Punkt zu Punkt. Gilt allerdings $r$ ×ース so ist der Schwingungsverlauf nahezu räumlich konstant.

Die numerische Auswertung von Gl. [13] erfordert Annahmen ïher $h, \mu, v, R, a$ und $a$. Näherungsweise kann man $\lambda=\mu$ setzen. Für die Geschwindigkeit in Diluvium kann man mit einiger Berechtigung $v=1,6 \mathrm{~m} / \mathrm{ms}$ annehmen. Die Grösse $R$ ist ron der Sprengstoffmenge ahhängig. Es ist schwer, für diesen Wert eine sichere Ahschät. zung zu erlangen, da wir noch zu wenig darüher wissen. Unter normaler Verhältnissen wird $R$ in einem Wertehereich von einigen decm bis zu wenigen m liegen. Es werde hier $R=1 \mathrm{~m}$ angenommen. Man erhät dann:

$$
\omega=1,51 \mathrm{~ms}^{-1}
$$

d. l.

$$
v=\frac{\omega}{2 \tau}=240 H_{z}
$$

und

$$
\varepsilon=1,067 \mathrm{~ms}^{-1}
$$

Infolge der Unsichrheit in der Amnahme von $R$, können (1) und $\varepsilon$ so- 
wohl halb so gross, wie auch doppelt so gross sein. Das Verhätnis (1)/E wird aljer durch diese Unsicherheit nicht berïhrt und beträgt

$$
\frac{\omega}{\varepsilon}=12
$$

wenn man nur $\hat{\imath}=u$ setzt. Das logarithmische Dekrement heträgt also

$$
\delta=4,44
$$

Noch unsicherer als $R$ ist eine Annahme über $\alpha$. Der schon zitierten Arlueit von Morris kann man entnehmen, dass a etwa von dersellıen Grossenordnungr wie e scin könnte. Da für den Fall $a=\varepsilon$ die Formel [13] besonders einfach wird, soll mit dieser Annahme zunäclsst grerechnet werden. Die Grösse a ist für die Zwecke dieser Untersuchung ohne Belang, da sie die Form der Schwingung nicht heeinflusst. Es werde nun noch $r>R$ angenommen und $t-\frac{r}{v}=t^{\prime}$ gesetzt. Dann ergilut sich die Bodenlsewegung aus [13]:

$$
\sigma_{\mathrm{r}}=\frac{a k^{\mathrm{o}}}{2 / \sqrt{\mu(\lambda+\mu)}(1)} \frac{e^{-\varepsilon t^{\prime}}}{r}\left[\sqrt{1,5} \sin \left(\omega t^{\prime}-0,96\right)+1-\varepsilon t^{\prime}\right]
$$

In Fig. 2 ist diese Schwiungung durch die ausgezogene Linie dargestcllt. Die gestrichelte Linie griht ein Bild vom zeitlichen Verlauf des Störimpulses $中(t)=a t e^{-\alpha t}$.

Un der Unsicherheit in derAnnahme von a Rechnung zu tragen, werde noch der extreme Grenzfall $\iota>>$ behandelt. In diesem Falle folgt aus [13]:

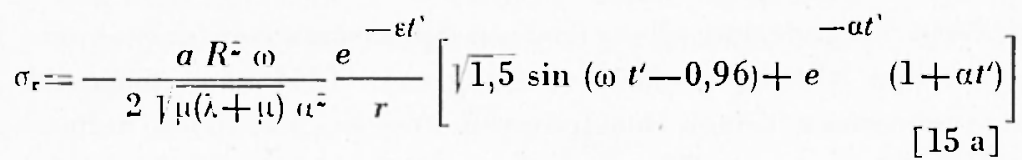

Der 2. Term in der eckigen Klammer von [15 a] ist nur für extrem kleine Werte von t' merklich. Alygeselsen rom allerersten Teil der Kurve, stellt [15 a] die reine Eigenschwingung dar: Die Behandlung des anderen Grenzfalles $u<<_{\varepsilon}$ ist nicht erforderlieh. Es ist unwahrscheinlich, dass der Störimpuls beim kritischen Radius $R$ lereits auf vicle ms auscinandergezogen ist.

Aus Fig. 2 und den Gleichungen [15] und [15 a] geht hervor, dass die Eigenfrequenz $v=\frac{\omega}{2 \pi}$ im primïren Spektrum der Explosions- 
welle vorherrschen muss. DieseFrequenz heträgt unter den hier gemachten Annahmen 240 1lz. Sicherlich sind diese Annahmen nicht gut hegrïndet, es ist aher kaum möglich, sie soweit abzuändern, dass die Eigenfrequenz unter $100 \mathrm{~Hz}_{z}$ liegt. Die in der Sprengseismik heohachteten Spektren werden also wahrscheinlich durch eine wesentliche Modifikation des primären Spektrums entstehen. Es kam bezweifelt werden, dass der in Vorhergehenden diskutierte Interferenzeffekt al-

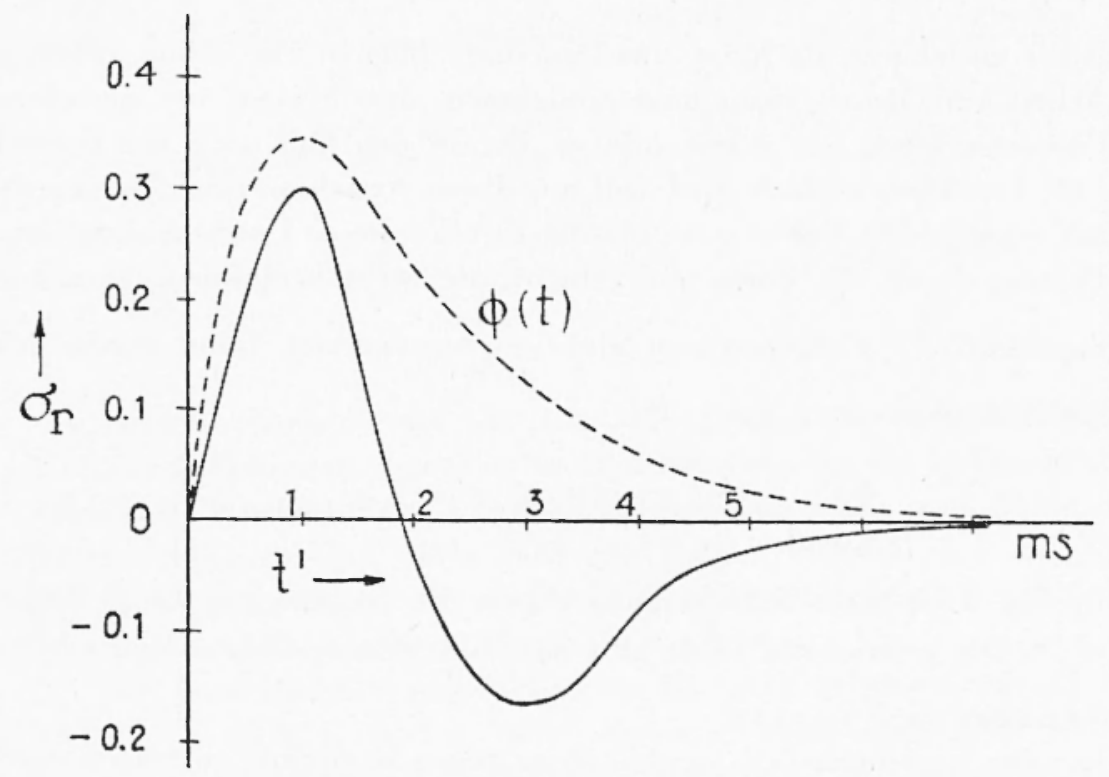

Fig. 2

lein diese Veränderung des primären Spektrums lewirken kann. Bei dem raschen Abklingen der Bewegung nach [15] kann man sich das Zustandekommen dieses Interfcrenzeffektes hei normalen Schusshohilochtiefen kaum vorstellen. Es müssen noch andere Effekte auftreten, welche erstens die holen Frequenzen hesonders stark schwächen, und zweitens eine Verlängerung des ganzen Impulses zur Folge hahen.

Kiirzlich haben Clewell und Simon $(*)$ eine Arheit veröffentlicht, in der die Anschauung vertreten wird, dass die Erdkruste wie ein Bandfilter wirkt. Die Schwächung der ticfen Frequenzen soll ein Interferenzeflekt sein, der dann eintritt, wenn die Wellenlänge gross ist gegeniiher der Dicke von Schichten, die in das homogene Medium eingelagert sind und sich von diesem durch Dichte und elastischen Konstanten unterscheiden. Das Auslöschen der holen Frequenzen wird 
nach Ansicht dieser Autoren, alogesehen ron der Viskosität, durch Streuung an kleinen Inhomogenitaten verursacht, wie bei der RayleighStreuung des Lichtes. An dem Vorhandensein derartiger diinner Schichten und Inhomogenitäten kamn kein Zweifel sein. Eine quantitative Alsschätzung ihres Einflusses auf durchgehende Wellen ist iiberaus schwierig. Für den Fall einer eingelagerten Schicht soll das unter der - allerdings sehr speziellen - Voraussetzungr versucht werden, dass die Wellennormale senkrecht auf den parallel angenommenen Schichtgrenzen steht.

Der Nullpunkt des Koordinatensystems werde in die obere Begrenzungseliene der eingelagerten Schicht grelegt. (s. Fig. 3.).

Die z-Achse zeige senkrecht nacl, unten. $d$ sei die Schichtdicke. Oherhall, und unterhall, der Schicht

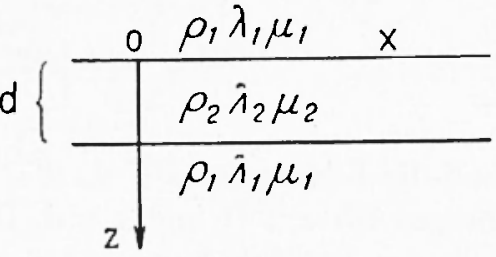

Fig. 3 sei $\because 1$ die Dichte $\rangle_{1}$ und $\|_{1}$ die Lamésclıen Konstanten. Die entsprechenten Werte in der Schicht seien $\varrho_{2}$, t., $!_{2}$. Senkrecht zur Ehene $z=O$ falle eine ehene longitudinale Welle ein. An der Einfallsebene tritt eine Aufteilung der Wellenenenergie cin. Fin Teil der Energie wird in das obere Medium reflektiert, ein anderer Teil dringt in die Schicht ein. An der Elsene $z=d$ crfolart dersellue Vorgang. Der von dieser Fläche reflektierte Energieanteil fillt von unten auf die Ebene $z=O$. wo wiederum Enerorieaufteilung stattfindet. Der Vorgang wieclerholt sicl, fortgesetzt. Von jeder der beiden Grenzlächen wird also nach oben und nach unten cine - wie man leicht sieht - konvergierende unendliche Reibe von Wellen alygestrahlt. die miteinander interferieren. Die einfallende Welle werde durch:

$$
u_{1}=A_{1} e^{i(1)\left(t-z a_{1}\right)}
$$

dargestellt, wobei $w_{1}$ die Verrükun (in der z-Richtungi und $a_{1}$ die Geschwindigkkeit im olıeren Medium ist. Yon der Fläiche $z=O$ wird nach oben die Welle

$$
\bar{w}_{1}=B_{1} e^{i \omega\left(t \div z_{1}, a_{1}\right)}
$$

und nach unten in die Schicht die Welle:

$$
u_{0}=A_{0} e^{i \omega\left(t-z_{1} a_{1}\right)}
$$


alogestrablt. Entsprechend lauft von der Ebene $z=d$ in die Schicht dic Welle:

$$
\bar{w}_{2}=B_{2} e^{i(1)\left(t+z^{\prime} a_{2}\right)}
$$

und nach unten die Welle

$$
w_{3}=A_{3}{ }^{i(1)\left(t-z a_{1}\right)}
$$

al. $\overline{w_{1}}, w_{2}, \bar{w}_{-}$und $w_{3}$ sind dabei Wellen, die sich durch die elsen beschriebene Interferenz ergelıen. Es gilt:

$$
a_{\mathrm{v}}=\sqrt[1]{\frac{\sqrt{\lambda_{\mathrm{v}}+2 \eta_{\mathrm{v}}}}{\rho_{\mathrm{v}}}} \quad(v=1,2)
$$

und die Konstanten $B_{1}, A_{2}, B_{2}, A_{3}$ berechnet man aus den Randluedingungen für $z=O$ und $z=d$. Die Randbedingungen verlangen Stetigrkeit der Verrïckungen und der Normalkomponenten des Spannungstensors. Man findet das Gleichungssystem:

$$
\begin{aligned}
& A_{1}+B_{1}=A_{2}+B_{2} \\
& \varrho_{1} a_{1}\left(A_{1}-B_{1}\right)=\varrho_{2} a_{9}\left(A_{2}-B_{2}\right) \\
& A_{2} e^{-i \frac{(1) d}{a_{2}}}+B_{2} e^{+i \frac{(1) d}{a_{2}}}=A_{3} e^{-i \frac{(1) d}{a_{1}}} \\
& \varrho_{2} a_{n}\left(A_{9} \cdot-i \frac{(1) d}{a_{2}}-B_{9} e^{\dagger i \frac{(1) d}{a_{2}}}=\varrho_{1} a_{1} \quad A_{3} e^{-i \frac{(1) d}{a_{1}}}\right.
\end{aligned}
$$

Setzt man zur Alskiirzuncr

$$
\frac{\varrho_{2} a_{2}}{\varrho_{1} a_{1}}=-P
$$

so erhält mañ die Lösungr:

$$
\begin{aligned}
& B_{1}=\frac{i\left(\frac{1}{p}-p\right) \sin \frac{(i) d}{a_{2}}}{2 \cos \frac{(1) d}{a_{2}}+i\left(\frac{1}{p}+p\right) \sin \frac{\omega d}{a_{2}}} A_{1} \\
& A_{3}=\frac{2 e^{i \frac{(1) d}{a_{1}}}}{2 \cos \frac{\omega d}{a_{2}}+i\left(\frac{1}{p}+p\right) \sin \frac{\omega d}{a_{2}}} A_{1}
\end{aligned}
$$

Die Konstanten $A_{2}$ und $B_{2}$ interessieren hier nicht.

Die Ausdrücke [16 a] his [ $16 \mathrm{e}$ ] stellen an sich noch keine wirk- 
lichen Wellen dar, da sie an jeder Stelle $z$ eine von $t=-\infty$ bis $t=\infty$ andauemde Schwingung hedeuten. Um zu wirklichen Wellen zu gelangen, muss man die Grössen $A_{1}, A_{2,}, A_{3,}, B_{1}$ und $B_{2}$, als Funktionen von (1) ansehen und die rechten Seiten derGleichungen [16 a] bis [16l1] ïher (1) von $-\infty$ bis $+\infty$ integrieren. Das ergibt:

$$
\begin{aligned}
& u_{1}=\int_{-\infty}^{+\infty} A((1)) e^{i(1)\left(t-z / a_{1}\right)} d(1) \\
& \bar{w}_{1}=\int_{-\infty}^{+\infty} A((\omega)) \frac{i\left(\frac{1}{p}-p\right) \sin \frac{\omega d}{a_{2}}}{2 \cos \frac{(1) d}{a_{2}}+i\left(\frac{1}{p}+p\right) \sin \frac{(\omega d}{a_{0}}} e^{i(\omega)\left(i-\alpha_{i} u_{1}\right)} d(1) \\
& w_{3}=\int_{-\infty}^{+\infty} A((1)) \frac{2 e^{i \frac{(1) d}{a_{1}}}}{2 \cos \frac{(1) d}{a_{2}}+i\left(\frac{1}{p}+p\right) \sin \frac{\omega d}{a_{2}}} e^{i(s)\left(t-z a_{1}\right)} d(1) \quad[21 c]
\end{aligned}
$$

Ulher die einfallende Welle werde folgende spezielle Annahme gemacht :

$$
\begin{aligned}
w_{1} & =0 & & t<z / a_{1} \\
u_{1}=e^{i \sigma\left(t-z / a_{1}\right)} & \text { für } & & t \geqslant z / a_{1}
\end{aligned}
$$

Dahei sei a komplex mit positivem Imaginärteil, und von den Funktionen $w_{1}, \bar{w}_{1}$ und $u_{3}$ möge nur der Imaginärteil physikalisch reellsein. Die Funktion $A(1)$ in den Gln. [21 a] - [2lc] lautet dann:

$$
f(11)=\frac{1}{2 \pi i} \frac{1}{(1)-\sigma}
$$

Durch Verschiehung des Integrationswegres kann man nun $\bar{w}_{1}$ und ":3 berechnen. Es ist:

$$
u_{3}=\frac{1}{\pi i} \int_{-\infty}^{+\infty} \frac{e^{i(\omega)\left(t-\frac{z-d}{a_{1}}\right)}}{2 \cos \frac{(1) d}{a_{2}}+i\left(\frac{1}{p}+p\right) \sin \frac{\omega d d}{a_{0}}} \frac{d(\omega)}{\omega-\sigma} \quad\left[21 \mathrm{c}^{*}\right]
$$

Die Pole des Integranden liegren bei

$$
\text { (1) }=\sigma
$$


und hei

$$
{ }_{(1)_{\mathrm{n}}}=\frac{a_{\mathrm{o}}}{d} \pi . n+i \frac{a_{\mathrm{o}}}{d} \operatorname{arctgh} \frac{2}{p+1 / p} \quad(n=0, \underline{-1},-2, \ldots)
$$

Sie hahen also alle einen positiven Imaginäteil. Daraus folgrt:

$$
\begin{aligned}
& w_{3}=0 \quad, \quad \text { für } t<\frac{z-d}{a_{1}} \\
& \left.w_{3}=\frac{2 e^{i \sigma\left(t-\frac{z-l}{a_{i}}\right)}}{2 \cos \sigma \frac{d}{a_{2}}+i\left(\frac{1}{p}+p\right) \sin \sigma \frac{d}{a_{2}}}-\frac{2 i}{p-1 / p}-i=1\right)^{n} \frac{\rho^{i(1)_{n}\left(t-\frac{z-d}{a_{1}}\right.}}{\left(1_{n}-\sigma\right.}, \\
& \text { für } t \geqslant \frac{z-d}{a_{1}}
\end{aligned}
$$

Bei dieserBerechnung ist $\sigma f\left(t_{n}\right.$ angenommen worden. Lis ist nicht schwierig, auch den Fall zu hehandeln, dass irgend ein $(1)_{n}$ mit $\sigma$ zusammenfällt. Doch hier soll darauf verzichtet werden.

Ganz entsprechend erfolgt die Auswertung des Integrales ron [21 h] Man sclureiht zunäiclıst:

$$
\begin{aligned}
\bar{w}_{1}=\frac{1 / p-p}{4 \pi i}[ & \int_{-\infty}^{+\infty} \frac{e^{i(1)\left(t+z\left(a_{1}+d_{1} a_{2}\right)\right.}}{2 \cos \frac{\omega d}{a_{0}}+i\left(\frac{1}{p}+p\right) \sin \frac{\omega d}{a_{0}}} \frac{d(1)}{\omega-\sigma}- \\
& \left.-\int_{-\infty}^{+\infty} \frac{e^{i \omega\left(t+z ; a_{1}-d / a_{2}\right)}}{2 \cos \frac{\omega d}{a_{n}}-i\left(\frac{1}{p}+p\right) \sin \frac{\omega d}{a_{0}}} \frac{d(1)}{\omega-\sigma}\right]
\end{aligned}
$$

Die Pole sind dieselhen wie bein Integral von [2l $\left.\mathrm{e}^{*}\right]$. Die Integration ergibt:

$$
\begin{aligned}
& \bar{w}_{2}=\frac{1}{2} \frac{\frac{1}{p}-p}{2 \cos \sigma \frac{d}{a_{2}}+i\left(\frac{1}{p}+p\right) \sin \sigma \frac{d}{a_{2}}}\left[e^{i \sigma\left(t+z a_{1}+d a_{2}\right)}-e^{\left.i \sigma(t+z)+a_{1}-d a_{2}\right)}\right]+ \\
& +\frac{i}{2} \sum_{\mathrm{n}=-\infty}^{+\infty} \frac{(-1)^{\mathrm{n}}}{\omega_{\mathrm{n}}-\sigma}\left[e^{i()_{\mathrm{n}}\left(t+z / a_{1}+d / a_{2}\right.}-e^{i \omega_{\mathrm{n}}\left(t+z a_{1}-d a_{2}\right)}\right]
\end{aligned}
$$


Bei nülherer Betraclitung dieser Lösungen erkennt man sofort, dass sie physikalische Engereimtheiten enthalten, welche ans der im mathematischen Ansatze enthaltenden Idealisierung folgen. So würde die Welle $w_{;}$von der Stelle $z=d$ hereits zur Zeit $t=O$ nach unten abliufen. Dies ist natiirlich unmöglich wenn die einfallende Welle zur Zeit $t=O$ am Orte $z=O$ eintrifft. Diese Diskrepanz ist eine Folge der Tatsache, dass im Ansatze alle Wellen simultan angenommen worden sind. Die Berechtigung dazu ist durch die Voratssetzungr gegehen, dass die Schichtdicke $d$ klein ist gegenïher der Wellenlänge der einfallenden Wellen in der Schicht. Die Lösung für

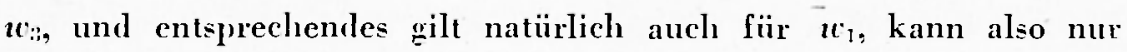
als eine Approximation angeselien werden, welche umso besser ist, je grösser der Wert $t-\frac{z-d}{a_{1}}$ ist, und die erst für solche Werte von $t-\frac{z-d}{a_{1}}$ brauchlbar ist. welche ein klcines Vielfaches von $d / a_{0}$ betragen. Im Falle der Welle $\boldsymbol{w}_{1}$ würden die durch die ersten Terme in den eckigen Klammern von [26] dargestellten Wellen zur Zeit $t=-\frac{z}{a_{1}}-\frac{d}{a_{2}}$ beginnen und die durch die zweitenTerme dieser

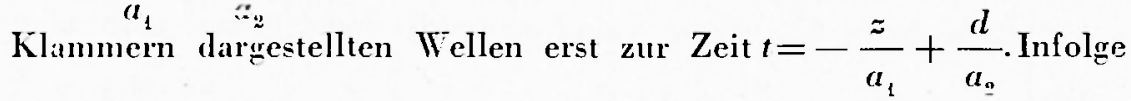
der elıen auseinandergesetzten Einschränkung̣ des Geltungshbereiches der Lösunø̣ kann man beide Wellen getrost superponieren und erhält:

$$
\begin{gathered}
\bar{w}_{1}=\frac{i\left(\frac{1}{p}-p\right) \sin \sigma \frac{d}{a_{2}}}{2 \cos \sigma \frac{d}{a_{2}}+i\left(\frac{1}{p}+p\right) \sin \sigma \frac{d}{a_{2}}} e^{\imath \sigma\left(t+z_{i} a_{1}\right)}- \\
-\sum_{n=-\infty}^{+\infty} \frac{(-1)^{\mathrm{n}}}{(1)_{\mathrm{n}}-\sigma} e^{i \omega_{\mathrm{n}}\left(t+z a_{1}\right.} \sin \left(\mathrm{t}_{\mathrm{n}} \frac{d}{a_{\mathrm{2}}}\right.
\end{gathered}
$$

Die Wellen $w_{3}$ und $\widetilde{w}_{1}$ hestehen aus 3 Anteilen. Der erste Anteil lat die Form der einfallenden Welle mit veründerter Amplitude und Plase. Der zweite Anteil ist unperiodisch $(n=O)$, der dritte Anteil ist eine gedämpfte Welle mit der Grundperiode $2 \frac{d}{a_{2}}$. Die Grössen (1) kann man als komplexe Eingenfrequenzen der durch die einfallende Welle angereg̣ten Schicht ansehen. Da der Realteil ron $\omega_{n}$ 
aher sehr gross ist, wird die Welle mit der Grundperiode $2 \frac{d}{a_{2}}$ zumindest hei $w_{3}$ keine wesentliche Rolle spielen. Bei $\bar{w}_{1}$ allerdings kamn infolge des geringen Ahsoluthetrages von $\sigma \frac{d}{a_{0}}$ der erste Anteil an Bedeutung verlieren. Dic Wirkung der Schicht wird also darin hestehen, dass die durchgehende Welle in ihrer Form nur geringfügrigg verändert wird. on der von der Schicht reflektierten Welle könncn die Eigenfrequenzen der Schicht eventucll cine Rolle spielen. In Figr. 4 ist die einfallende und dic durchgehende Welle für cinen speziellen Fall zur Illustration des Gesarten dargestellt worden Dic numerischen Annahmen sind dahei:

$$
\begin{aligned}
& a_{1}=1,6 \mathrm{~m} / \mathrm{ms} \quad ; \quad a_{0}=2,4 \mathrm{~m} / \mathrm{ms} \quad ; \quad p=1,5 \quad ; \quad d=1 \mathrm{~m} \\
& \sigma=(1.51+1,07 i) \mathrm{ms}^{-1}
\end{aligned}
$$

Bei sehr kleinen Schichtdicken wird also, zumindest hei senkrechtem Welleneinfall, keine sehr merkliche Anderung des Spektrums au er-

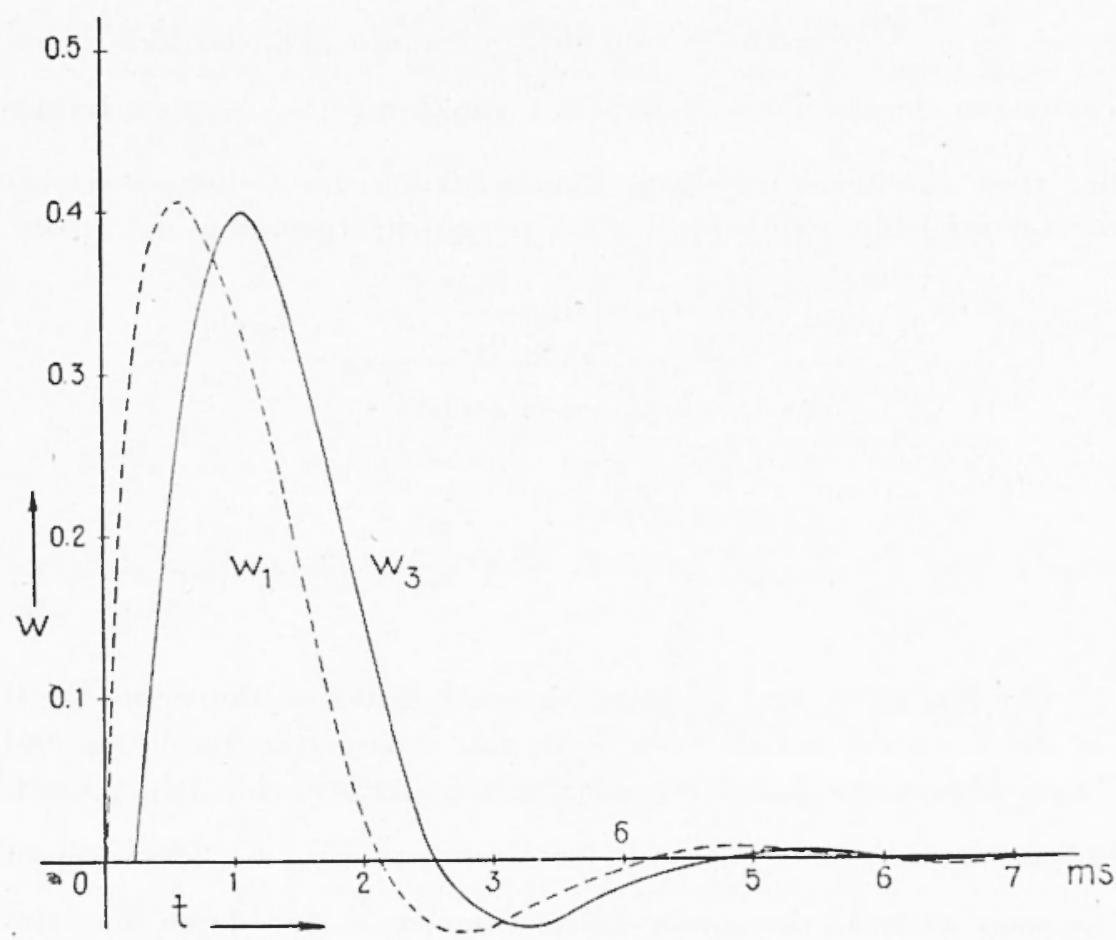

Fig. 4 
warten sein. In entgegengesetzten Grenfalle einer Schichttlicke, die sehr gross gegeniilher der Wcllenlïng ist, kann man üherhaupt keine Umwandlung des Spektrums annelimen Bevor es zu einer Interferenz zwischen zwei Wellen kommen kann, ist die erste hereits praktisch verschwunden. Der Fall, dass die Wellenlänge in der Schicht und die Schichtdicke ron etwa gleicher Grössenordnung ist, kann mit der im Vorhergehenden angewandten Methode nicht erledigt werden. Die Wirkungr einer derartigen Schicht auf eine durchgehende Welle soll hier nicht behandelt werden, da eine mehrere Meter mächtige Einlagrerung von merklich verschiedener Schallhärte im tieferen Untergrunde nicht so häufig rorkommen diirfte, dass man das als einen Normalfall anselien könntc. Dagegen ist der Fall, dass Schichtmäehtigkeit und Wellenlïngre von merklich gleicher Grössenordnung sind, fiir den Fall des Einfallens einer Welle an der Erdoherflüche von Bedeutung. Nelunen wir einmal an, dass die Wellengeschwindirkeit in der sog. Verwitterungsschicht $0.8 \mathrm{~m} / \mathrm{ms}$ ist. Einell elle, in der eine Frequenz von $2.10 \mathrm{~Hz}$ vorherrscht, wird in dieser Schicht eine Wellenlïnge von $3.33 \mathrm{~m}$ hahen. Es ist hekannt, dass die Dicke der Verwitterungsschicht von gleicher Grössenordnung ist. Es soll daher nun die

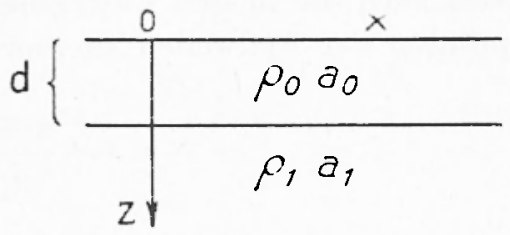

Fig. 5

Theorie der Reflexion für eine senkrecht auf eine geschichtete Erdoherfliche einfallende Welle betrachtet werden.

Die Ebene $z=O$ sei die Erdoberfläche, und die Elene $z=d$ sei. die untere Begrenzung der Verwitterungsschicht, die von gleicher Dicke und bomogen angenommen werde. $\varrho_{0}, a_{0}$, beznw. $\varrho_{1}, a_{1}$ seien Dichte und Wellengeschwindigkeit in der Verwitterungsschicht bzw. im bomogenen I'ntergrunde. In der Flïche $z=d$ falle zur Zeit $t=0$ die Welle $u_{1}$ von unten ber ein. Sie wird in cine reflektierte Welle $\bar{w}_{1}$ und in eine durchgehende Welle $w_{0}$ aufgespalten. Zur Zeit $t=\frac{d}{a_{0}}$ 
trifft $"$ auf die Fläche $z=O$. Die von dieser Fläche reflektierte Welle sei $\bar{w}_{0}$, welche zur Zeit $t=\frac{2 d}{a_{0}}$ von oben her auf die untere Grenzfläche trifft. Die dahei durch Reflexion und Brechung entstehenden Wellen $w_{0}^{(1)}$ bzw. $\bar{w}_{1}^{(1)}$. Diese Vorgänge wiederholen sich nun unendlich oft, wobei aler die Energie der Teilwellen nach einer endlichen Zahl von Reflexionen praktisch bereits unmerklich wird. Betrachten wir zunächst die Vorgünge an der Ebene $z=O$. Die von dieser Fläche durch Interferenz der einzelnen Teilwellen zu beobachtende Schwingung sei $\zeta_{\text {.. }}$ Dann ist:

$$
\begin{aligned}
& \varlimsup_{0}=0 \\
& \text { fïr }-\infty<t<\frac{d}{a_{0}} \\
& \zeta_{0}=\left[w_{0}+\bar{w}_{0}\right]_{z=1} \\
& , \quad \frac{d}{a_{0}} \leqslant l<3 \frac{d}{a_{0}} \\
& \zeta_{0}=\left[w_{0}+\bar{w}_{0}+\sum_{\mathrm{v}=1}^{\mathrm{n}}\left(w^{(v)}+\bar{w}_{0}^{(v)}\right)\right]_{z=0} \quad, \quad(2 n+1) \frac{d}{a_{0}}<t<(2 n+3) \frac{d}{a_{0}}
\end{aligned}
$$

Entsprechend setzt sich auch die in den Untergrund reflektierte Welle $W_{1}$ durch Superposition der Teilwellen zusammen. Es gilt:

$$
\begin{aligned}
& \ddot{W}_{1}=0 \\
& W_{1}=\bar{w}_{1} \\
& W_{1}=\bar{w}_{1}+\bar{w}_{1}^{(1)} \\
& -\infty<t-\frac{z-d}{a_{1}}<0 \\
& 0 \leqslant t-\frac{z-d}{a_{1}}<2 \frac{d}{a_{0}} \\
& \text { für } \\
& 2 \frac{d}{a_{0}}-t-\frac{z-d}{a_{1}}<4 \frac{d}{a_{0}} \\
& W_{1}=\bar{w}_{1}+\sum_{s=1}^{n} \bar{w}_{1}^{(v)} \\
& 2 n \frac{d}{a_{n}}<t-\frac{z-d}{a_{4}}<2(n+1) \frac{d}{a_{0}}
\end{aligned}
$$

Setzt man ein partikuläres Integral für die einfallende Welle in der Form:

$$
w_{1}=A(\omega) e^{i \omega\left(t+z a_{1}\right)}
$$


an, so liefert die Reflexionstheorie die partikulären Lösungen:

$$
\begin{aligned}
& w_{0}=\frac{2 p}{p+1} A((1)) e^{i \omega\left(1+\frac{z-d}{a_{0}} \div \frac{d}{a_{1}}\right)} \\
& \bar{\psi}_{0}=\frac{2 p}{p+1} A(\omega) e^{i(1)\left(t-\frac{z+d}{a_{0}}+\frac{d}{a_{1}}\right)} \\
& \bar{w}_{1}=\frac{p-1}{p+1} A((1)) e^{i(1)\left(t-\frac{z-2 d}{a_{1}}\right)} \\
& \left.w^{(v)}=\frac{2 p}{p+1}\left(\frac{1-p}{1+p}\right)^{v} A((1)) e^{i(1) l t+\frac{z-(2 v+1) d}{a_{0}}}+\frac{d}{a_{1}}\right] \\
& \bar{u}^{(v)}=\frac{2 p}{p+1}\left(\frac{1-p}{1+p}\right)^{v} A((1)) e^{i \omega\left[t-\frac{z+(2 v+1) d}{a_{0}}+\frac{d}{a_{1}}\right]} \\
& \bar{w}^{(v)}=\frac{4 p}{(1+p)^{2}}\left(\frac{1-p}{1+p}\right): A(n) e^{\left.i(1) j i-\frac{z-2 d}{a_{1}}-\frac{2 v d}{a_{0}}\right]}
\end{aligned}
$$

Die Welle $u_{1}$ soll nun ebenso speziell angenommen werden wie auf S. 10. Es sei also:

$$
\begin{aligned}
& w_{1}=0 \\
& w_{1}=e^{i \sigma\left(t+z a_{1}\right)} \quad \text { für } \quad t=-\frac{z}{a_{1}}+\frac{d}{a_{1}}
\end{aligned}
$$

Dann wird:

$$
A(\omega)=\frac{1}{2 \pi i} \frac{e^{-i(1) d a_{1}}}{(1)-\sigma}
$$

Im Intervall $\left.(2 n+1) \frac{d}{a_{n}} \leqslant t<(2)^{\prime}+3\right) \frac{d}{a_{0}}$ erhält man für $i_{0}$ :

$\zeta_{0}=\frac{1}{2 \pi i} \frac{4 p}{1+p} \int_{-\infty}^{+\infty} \frac{\left(\frac{1-p}{1+p}\right)^{n+1} e^{-2 i(1)(n+1) d / a_{0}}-1}{\frac{1-p}{1+p} e^{-2 i \omega u_{0}-1}} e^{i(1)\left(t-d a_{0}\right)} \frac{d(1)}{(1)-\sigma}, n \geqslant 1$

Um dieses Integral durch Integration über einen geeigneten komplexen Weg auszuwerten, teilt man es in zwei Teile auf: 


$$
\begin{aligned}
\zeta_{0}= & \frac{1}{2 \pi i} \frac{4 p}{p+1} \int_{-\infty}^{+\infty} \frac{\left(\frac{1-p}{1+p}\right)^{n+1}}{\frac{1-p}{1+p} e^{-2 i(1) d / a_{0}}-1} e^{i \omega\left[t-(2 n+3) d a_{0}\right]} \frac{d_{(1)}}{(1)-\sigma}- \\
& -\frac{1}{2 \pi i} \frac{4 p}{p+1} \int_{-\infty}^{+\infty} \frac{1}{\frac{1-p}{1+p} e^{-2 i(1) d / a_{0}}-1} e^{i(0) d\left(t-d / a_{0}\right)} \frac{d(1)}{(1)-\sigma}
\end{aligned}
$$

Fiir $t<(2 n+3) d / a_{0}$ verseliwindet der erste Term von [32']. ¿ wird also in dem betrachteten Intervall nur durch den zweiten, iiberhaupt nicht mehr von n abhängigen, Term dargestellt. Zur Auswertung dieses zweiten Integrales nuss man zumächst die Pole des Integranden berechnen. Ausser bei $\omega=\sigma$ liegen diese hei.

$$
(1)_{\mathrm{r}}=(2 r+1) \frac{a_{0}}{2 d} \pi+i \frac{a_{0}}{2 d} \ln \frac{p+1}{p-1} \quad, \quad(r=0, \pm 1,-2, \ldots)[33]
$$

Integriert man nun um die Pole, so crhält man:

$$
\begin{aligned}
\tau_{0}= & -\frac{4 p}{1+p} \frac{1}{\frac{1-p}{1+p}-2^{2 \sigma \sigma d_{i}\left(a_{0}\right.}-1} e^{i \sigma\left(t-d a_{0}\right)} \\
& +i \frac{a_{0}}{d} \frac{2 p}{p-1} \sum_{\mathrm{r}=-\infty}^{+\infty} \frac{1}{(1)_{\mathrm{r}}-\sigma} e^{i()_{\mathrm{r}}\left(t+d a_{0}\right)}, \quad \sigma \neq=()_{\mathrm{r}}
\end{aligned}
$$

und diese Lösung grilt fiir:

$$
3 \frac{d}{a_{0}} \leqslant \ell<x
$$

die Gl. [32"] ist zu ergänzen durch:

$$
\check{\varsigma}_{0}=0 \quad \text { für } \quad t<d / a_{0}
$$

und durch

$$
\varsigma_{0}=\frac{4 p}{p+1} e^{i \sigma\left(t-d a_{0}\right)} \quad \text { für } \quad \frac{d}{a_{0}}<t<\frac{3 d}{a_{0}}
$$

Auf ganz entsprechende Weise lïsst sich dic Welle $\Pi_{1}$ berechnen. Zunäichst erhült man für $2 n d / a_{0} \leqslant 1-\frac{z-d}{a_{1}}<2(n+1) d / a_{0}$ 


$$
W_{1}=\frac{1}{2 \pi i} i_{\infty}^{+\infty}\left(\frac{p-1}{p+1}+\frac{4 p}{(p+1)^{2}} \sum_{v=1}^{n}\left(\frac{1-p}{1+p}\right)^{v-1}=2 i(1) v d a_{2}\right) i(1)\left(t-\frac{z-d}{a_{1}}\right) \frac{d(1)}{(1)-\sigma}
$$

Nach Umrechununy, findet man:

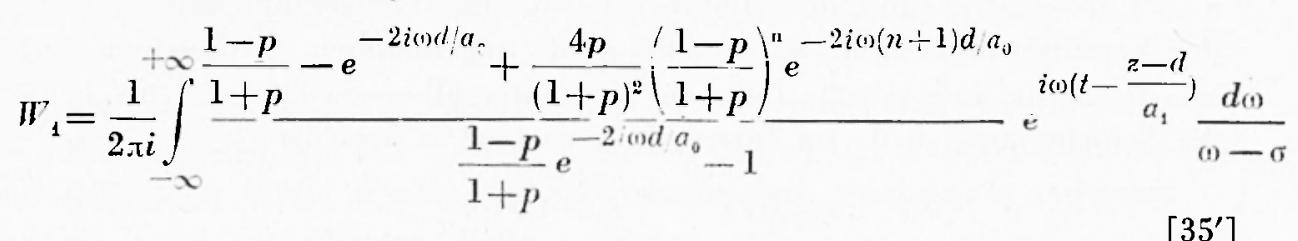

Spaltet man auch dieses Integral wie das von Gl. [32] in Teilintegrale auf, so ist in dem hetrachteten Interral das Teilintegral, welches dem 3. Summanden des Zählers in [35'] entspricht, Null. Die Pole werden wieder durch [33] gegehen, und die Integration führt auf die Lösung:

$$
\begin{gathered}
W_{1}=0 \quad, \quad \text { für } t<\frac{z-d}{a_{1}} \\
W_{1}=\frac{p-1}{p+1} e^{i \sigma\left(t-\frac{z-d}{a_{1}}\right.} \text { für } \frac{z-d}{a_{1}} \leqslant t<\frac{z-d}{a_{1}}+2 \frac{d}{a_{0}}\left[35^{* *}\right] \\
W_{1}=\frac{\frac{1-p}{1+p}-e^{-2 i \sigma d a_{0}}}{\frac{1-p}{1+p} e^{-2 \ell \frac{\pi}{1+l} a_{0}}-1} e^{\left.i \sigma t-\frac{z-d}{a_{1}}\right)}
\end{gathered}
$$

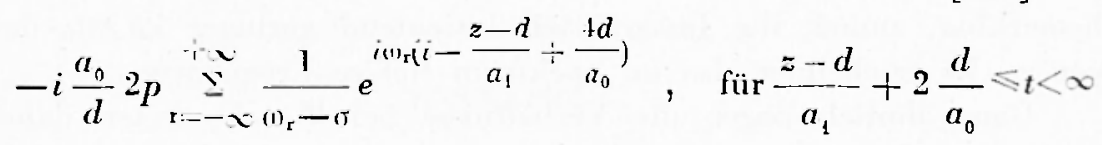

Das Auftreten der Eigenfrequenzen $\omega_{r}$ ist eine Folge der Tatsache, dass an den Grenzflächen $z=O$ und $z=d$ periodisch neue Energieaufteilungen vor sicl, gehen. Die Grundperiode ist daluei $T_{0}=4 d / a_{0}$ Die Eigenfrequenzen müssen komplex sein, weil die verfürhare Energie nicht unendlich gross ist. Man könnte die Vorgänge natürlich auch durch Wellen heschreilien, in denen nur die Frequenz o auftritt. Bei einer derartigen Beschreilung aher, wiurden in jedem dex Intervalle $(2 n+1) \frac{d}{a_{0}} \leqslant t<(2 n+3) \frac{d}{a_{0}}$ die Amplituden und Phasen 
ron $n$ abhängig sein. In dieser Veränderungr der Amplituden und Plasen mit dem Intervall drückt sich grerade das Vorkommen der Eigenfrequenzen (1) aus

$T_{m}$ einen anschaulichen Einblick in die Verhältnisse zu grewinnen, seien einige numerische Rechmungen angefügt: Wie auf $S$. 13 sei $\sigma=1,51+1,07 i$ und $n_{1}=1,6 \mathrm{~m} / \mathrm{ms}$. Für die Geschwindigkeit in der Verwitterungsschicht sei $a_{0}=0.8 \mathrm{~m} / \mathrm{ms}$ angenommen. Schliesslich sei $d=2 \mathrm{~m}$ und $p=2$. Dann ist im Intervall $-\infty<t<2,5 \mathrm{~ms}$, die Schwingung $\zeta_{0}=0$. In Intervall $0 \leqslant \tau=t-2,5<5 \mathrm{~ms}$ ist:

$$
\zeta_{0}=2,67 e^{-1.07 \tau} \sin 1,51 \tau
$$

In Intervall $0 \leqslant \tau^{\prime}=t-7,5<\infty$ besteht $\bigodot_{0}$ aus einem Anteile

$$
\check{c}_{0}^{(1)}=-1,84.10^{-4},-1.0 \tau \tau^{\prime} \sin \left(1,51 \tau^{\prime}+2,55\right)
$$

und einem Anteil ${ }^{(2)}$. welcher eine gedimpte periodische Schwingungr darstellt. Die Grundperiode von $\overbrace{}^{-(2)}$ beträgt $10 \mathrm{~ms}$. Die Perioden der Oberschwingungen ergelocn sich aus $T_{\mathrm{r}}=10 /(2 r+1)$ ms. Dic Dämpfungskonstante ist für die Grundschwingung und alle Oberschwingungen gleich gross und hat den Wert 0,22 ns $^{-1}$ Für die Grundsclowingung crhält man:

$$
\tau_{00}^{(2)}=0,22 e^{-0.22 \tau^{\prime}} \cos \left(0,63 \mathrm{r}^{\prime}-0,63\right)
$$

Die Amplituden der Oberschwingungen nelumen nur selır langsam mit wachsendem $r$ al, sodass die löheren Frequenzen das Bild der Schwingungr noch entscheidend beeinflussen.. Wichtiog ist, dass der An-

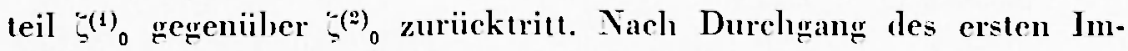
pulses in den ersten $5 \mathrm{~ms}$ machen sich die Eigenfrequenzen stark bemerkbar, zumal ihr Imarinarteil bedeutend geringer ist als der von $\sigma$. Fs erscheinen also im Spektrum tiefere Frequenzen.

Ganz abnlich liegen die Verhältnisse bei $I_{1}$. Es treten dabei dieselben Eigenfrequenzen auf wie bei $\varsigma_{0} . W_{1}$ ist Null für $t<\frac{z-d}{a_{1}}$ In Intervall $\frac{z-d}{a_{1}} \leqslant t<\frac{z-d}{a_{1}}+5$ ms erhält man:

$$
\Pi_{1}=0,33 e^{-1.0 \tau\left(t-\frac{z-d}{a_{1}}\right)} \sin 1,51\left(t-\frac{z-d}{a_{1}}\right)
$$

In Intervall $\frac{z-d}{a_{1}} \leqslant \tau=1-5<2$ beträint der erete Anteil ron $\Pi_{1}$ 


$$
W_{1}^{(1)}=1,44.10^{-2} \epsilon^{-1,07}\left(\tau-\frac{z-d}{a_{1}}\right) \sin \left[1,51\left(\tau \frac{z-d}{a_{1}}\right)+1,28\right]
$$

Beim zweiten Anteil beträgt der Beitrag der Welle mit der Grundperiode $T_{0}$ :

$$
W_{10}^{(2)}=0,07 e^{-0.22}\left(\mathrm{r}-\frac{z-d}{a_{1}}\right)\left(\operatorname { c o s } \left[0,63\left(\tau-\frac{z-d}{a_{1}} ;-0,63\right]\right.\right.
$$

Man sieht, dass bei $W_{1}$ die Eigenfrequenzen nicht eine ebenso grosse Rolle spielen wie hei $\zeta_{0}$, aher auch hei der an der Erdoberfläche reflektierten Welle werden im Spektrum tiefere Frequenzen auftreten als in der einfallenden Welle.

Es hat den Anschein, als ol, der auf Seite 2 hesprochene Interferenzeffekt im Zusammenwirken mit dem zuletzt diskutierten Effekt der geschichteten Oberfläche wesentlich das Spektrum der sprengseismischen Wellen hestimmt. Auch hei natürlichen Erdhebenwellen dürften die Vorgänge heim Einfall in die geschichtete Erdoherflä. che fiir das Spektrum dieser Wellen ron Bedeutung sein. Natürlic ${ }^{x}$ ist es crforderlich, die Untersuchung auf den Fall nicht senkrechter Inzidenz auszudehnen.

\section{ZUSAMMENFASSUNG}

Es werden einige Interferenzeffehte dishutiert, welche in einem ideal elastischen Medium das Spetitrum sprengseimischer Tiellen verädern. Der Einfluss der sog. Veruitterungszone scheint am bedeutendsten zu sein.

\section{LITERATLR}

1) Monris Gronge: Geophysics. Vol. XV, N. 1, 1950.

(-) Clewfl, D. H. and Sinox R. F.: Geophysics. Vol. XV, Nr. 1, 1950. 